\title{
Some results on the power graphs of finite groups
}

\author{
Doostabadi Alireza, Erfanian Ahmad*, Jafarzadeh Abbas \\ Ferdowsi University of Mashhad, \\ Department of Mathematics and Centre of Excellence in Analysis on Algebraic Structures, Mashhad, Iran
}

*Corresponding author, e-mail: erfanian@math.um.ac.ir

Received 24 Apr 2013

Accepted 21 Feb 2014

\begin{abstract}
We classify planar graphs and complete power graphs of groups and show that the only infinite group with a complete power graph is the Prüfer group $\mathbb{Z}_{p} \infty$. Clique and chromatic numbers and the automorphism group of power graphs are investigated. We also prove that the reduced power graph of a group $G$ is regular if and only if $G$ is a cyclic $p$-group or $\exp (G)=p$ for some prime number $p$.
\end{abstract}

KEYWORDS: reduced power graph, planar graph, perfect graph, regular graph, automorphism group of a graph

\section{INTRODUCTION}

For a semigroup or group, we can associate a graph in different ways to describe algebraic structures of the group or semigroup using the properties of graph. Bosak ${ }^{1}$ studied certain graphs over semigroups. The directed power graph of a semigroup was defined by Kelarev and Quinn ${ }^{2,3}$. Recently, Chakrabarty, Ghosh and Sen ${ }^{4}$ investigated the power graph of semigroups and characterized the class of semigroups with a connected or complete power graph. Also, Cameron ${ }^{5}$ proved that for finite groups, the undirected power graph determines the directed power graph up to an isomorphism and two finite groups with isomorphic undirected power graphs have the same number of elements of each order.

For a given group $G$ we may define the directed power graph $\vec{P}(G)$ as a directed graph with vertex set $G$ in which there is an arc from $x$ to $y$ if $x \neq y$ and $y=x^{m}$ for some positive integer $m$. The indegree (outdegree), denoted by indeg $\overrightarrow{\vec{\Gamma}}(u)$ (respectively, outdeg $\vec{\Gamma}_{\vec{\Gamma}}(u)$ ) of a vertex $u$ in a directed graph $\vec{\Gamma}$ is the number of arcs $(v, u)$ (respectively, $(u, v))$ in $\vec{\Gamma}$. The undirected power graph $\mathscr{P}(G)$ is defined in such a way that two distinct vertices $x$ and $y$ are adjacent if one of them is a power of the other one. For any graph $\Gamma$, we denote the set of vertices and the edges of $\Gamma$ by $V(\Gamma)$ and $E(\Gamma)$, respectively. If $X \subseteq G$ then we can also define $\mathscr{P}(X)$ as the induced subgraph on $X$ of $\mathscr{P}(G)$. Moreover, $|\Gamma|$ stands for the number of vertices of $\Gamma$ and is called the order of $\Gamma$. Two distinct vertices $v_{1}, v_{2}$ are adjacent if they are joined by an edge in $\Gamma$ and we denote this by $v_{1} \sim v_{2}$. The complement graph
$\bar{\Gamma}$ is defined as $V(\bar{\Gamma})=V(\Gamma)$ and two vertices are adjacent in $\bar{\Gamma}$ if and only if they are not adjacent in $\Gamma$. A proper colouring of a graph $\Gamma$ is a map from $V(\Gamma)$ into some finite set of colours such that no two adjacent vertices are assigned the same colour. If $\Gamma$ can be properly coloured with a set of $k$ colours, then we say that $\Gamma$ can be properly $k$-coloured. The minimum value of $k$ for which $\Gamma$ can be $k$-coloured is called the chromatic number of $\Gamma$ and we denote it by $\chi(\Gamma)$. The degree of vertex $v$ in $\Gamma$ is the number

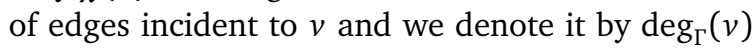
or simply $\operatorname{deg}(v)$. A graph automorphism $\varphi$ is a permutation on $V(\Gamma)$ such that $u, v$ are adjacent if and only if $\varphi(u), \varphi(v)$ are adjacent. The set of all graph automorphisms of $\Gamma$ is denoted by $\operatorname{Aut}(\Gamma)$. A clique is an induced subgraph of $\Gamma$ that is complete. The maximum order of a clique in $\Gamma$ is called the clique number of $\Gamma$ and is denoted by $\omega(\Gamma)$.

\section{PLANAR AND COMPLETE POWER GRAPHS}

In this section, we shall characterize all groups with a complete or planar power graph.

Definition 1 A group $G$ is called torsion group if the order all of elements of $G$ is finite.

Lemma 1 Let $G$ be a torsion group. If $x$ and $y$ are adjacent vertices in $\mathscr{P}(G)$, then either $|x|$ divides $|y|$ or $|y|$ divides $|x|$. The converse is true whenever $G$ is a cyclic group.

For a finite nontrivial group $G$, Chakrabarty et al proved that $\mathscr{P}(G)$ is complete if and only if $G$ is a cyclic group of prime power order ${ }^{4}$. In the next theorem, we investigate a result for infinite groups. Recall that a group $G$ is called locally cyclic 
if every finitely generated subgroup of $G$ is cyclic. Equivalently, $G$ is locally cyclic if $\langle x, y\rangle$ is cyclic for every pair $x, y$ of elements of $G$. In particular, every locally cyclic group is abelian. It is a well-known result that a group is locally cyclic if and only if it is isomorphic to a subgroup of $\mathbb{Q}$ or $\mathbb{Q} / \mathbb{Z}$ (see Ref. 6).

Definition 2 The power graph $\mathscr{P}(G)$ of a group $G$, is the graph whose vertex set is the group $G$ such that two distinct elements are adjacent if one is a power of the other.

Theorem 1 Let $G$ be an infinite group. Then $\mathscr{P}(G)$ is complete if and only if $G \cong \mathbb{Z}_{p^{\infty}}$ for some prime $p$.

Proof: Since every proper subgroup of the Prüfer group $\mathbb{Z}_{p^{\infty}}$ is a finite cyclic $p$-group, for every pair of elements of $G$, one is a power of the other, which implies that $\mathscr{P}\left(\mathbb{Z}_{p^{\infty}}\right)$ is an infinite complete graph.

Now suppose that $\mathscr{P}(G)$ is a complete graph for some infinite group $G$. Clearly $G$ is an abelian torsion group, which is also a $p$-group. Since $\mathscr{P}(G)$ is a complete graph, every pair $x, y$ of $G$ generates a cyclic subgroup. Hence $G$ is a locally cyclic $p$-group, which implies $G \cong \mathbb{Z}_{p^{\infty}}$.

In the following theorem, we give a necessary and sufficient condition for the power graph $\mathscr{P}(G)$ to be planar. Recall that by a well-known theorem of Kuratowski, a graph $\Gamma$ is planar if it has no subdivision of the graphs $K_{3,3}$ and $K_{5}$ (see Ref. 7).

Theorem 2 Let $G$ be a group. Then $\mathscr{P}(G)$ is planar if and only if $G$ is a torsion group and $\pi_{e}(G) \subseteq$ $\{1,2,3,4\}$, where $\pi_{e}(G)=\{|x|: x \in G\}$.

Proof: First assume that $\mathscr{P}(G)$ is a planar graph. We claim that $G$ is torsion group. If $G$ has an element of infinite order, then we can easily find subgraphs $K_{3,3}$ or $K_{5}$ in $\mathscr{P}(G)$, which is a contradiction. Now, suppose on the contrary that there exists an element $x \in G$ such that $|x| \geqslant 5$. If $|x|$ is divisible by a prime $p \geqslant 5$, then we have $\mathscr{P}\left(\left\langle x^{|x| / p}\right\rangle\right) \cong K_{p}$ which is impossible. Thus $|x|=2^{m} 3^{n}$, where $m$ and $n$ are non-negative integers. If $n=0, m \geqslant 3$ or $m=0$, $n \geqslant 2$, then $\mathscr{P}(\langle x\rangle) \cong K_{2^{m}}$ or $K_{3^{n}}$. Hence $\mathscr{P}(G)$ contains a subgraph isomorphic to $K_{5}$. Hence we may assume that $m, n \geqslant 1$ and 6 divides $|x|$ and consequently $\mathscr{P}\left(\left\langle x^{|x| / 6}\right\rangle\right)$ has a subgraph isomorphic to $K_{3,3}$, which is again a contradiction. Hence $\pi_{e}(G) \subseteq\{1,2,3,4\}$. Conversely, assume that $G$ is a torsion group and $\pi_{e}(G) \subseteq\{1,2,3,4\}$. If $x$ is an element of order 3 , then it is not adjacent to any element of order 2 or 4 by Lemma 1 . Therefore $\mathscr{P}(\langle x\rangle) \cong K_{3}$. Put $A=\{g \in G:|g|=3\}$. Then

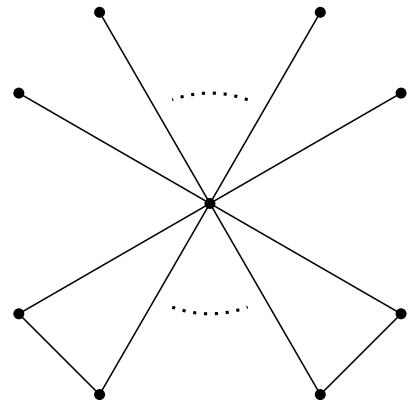

Fig. $1 \exp (G \backslash A)=2$.

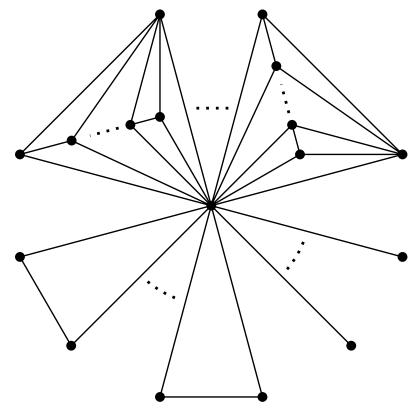

Fig. $2 \exp (G \backslash A)=4$.

$\exp (G \backslash A)=2$ or 4 . If $\exp (G \backslash A)=2$, then $\mathscr{P}(G \backslash A)$ is a star graph and so $\mathscr{P}(G)$ is a graph containing some triangles and the above star graph with the same identity (Fig. 1).

Now suppose that $\exp (G \backslash A)=4$. Consider $B=\{g \in G:|g|=4\}, C=\{g \in G:|g|=2\}$ and $D=C \backslash B^{\{2\}}$, where $B^{\{2\}}=\left\{g^{2}: g \in B\right\}$. For each $g \in B^{\{2\}}$, put $B_{g}=\left\{g^{\prime} \in B: g^{\prime 2}=g\right\}$. Then we can easily see that $\mathscr{P}\left(B_{g} \cup\{1, g\}\right)$ consists of some (not necessarily finitely many) subgraphs $K_{4}$ with two common vertices, namely the identity and $g$, and two non-common vertices $g^{\prime}, g^{\prime-1} \in B_{g}$. Thus $\mathscr{P}(G)$ involves a star graph with the identity as midpoint, some triangles and some complete graphs $K_{4}$ as above, all of them having the identity in common. Hence $\mathscr{P}(G)$ is planar (Fig. 2).

Corollary 1 If $G$ is a group with a planar power graph, then $\chi(\mathscr{P}(G))=\max \pi_{e}(G)$.

Proof: If $\mathscr{P}(G)$ is planar, then by Theorem 2, $\max \pi_{e}(G) \leqslant 4$. If $\max \pi_{e}(G)=4$, then $\mathscr{P}(G)$ is a graph as in Fig. 2 with a complete subgraph of order 4 , which implies that $\chi(\mathscr{P}(G))=4$. If $\max \pi_{e}(G)=3$, then $\mathscr{P}(G)$ contains complete subgraphs of order 3 that have the identity in common. Thus $\chi(\mathscr{P}(G))=\max \pi_{e}(G)=3$. In the case 
$\max \pi_{e}(G)=2, \mathscr{P}(G)$ is a star graph and clearly $\chi(\mathscr{P}(G))=2$.

As a direct consequence of the above theorem, one can deduce that the subgroups and quotients of a group with a planar power graph have planar power graphs. The following theorem is a consequence of a theorem given in Ref. 8 which determines the structure of all groups whose power graphs are planar.

Theorem 3 Let $G$ be a group. Then $\mathscr{P}(G)$ is planar if and only if $G$ is locally finite and has one of the following structures.

(i) $G$ is a group of exponent 2, 3, or 4 .

(ii) There is a normal elementary abelian 3-subgroup $N$ of $G$ such that $G / N$ is isomorphic to a subgroup of $Q_{8}$.

(iii) There is a normal elementary abelian 2-subgroup $N$ of $G$ such that $G=N S$ where $S \cong S_{3}$.

(iv) There is a normal 2-subgroup $N$ of $G$ of nilpotency class 2 such that $|G / N|=3$.

Example 1 For the quaternion group $Q_{8}$, we can see that its power graph is planar and it is clear that $Q_{8}$ is a group of exponent 4 .

\section{REGULARITY OF A POWER GRAPH WITHOUT IDENTITY ELEMENT}

The graph $\Gamma$ is regular if the all vertices have the same degree. We know that for a nontrivial finite group $G$ of order $n$ the degree of the identity element in the graph $\mathscr{P}(G)$ is $n-1$. Thus $\mathscr{P}(G)$ is a regular graph if and only if $\mathscr{P}(G)$ is a complete graph $K_{n-1}$. It is known that the power graph of $G$ is complete if and only if $G$ is a finite cyclic $p$-group, where $p$ is prime. The reduced power graph of a finite group $G$ is obtained when we remove the identity element from the vertex set and is denoted by $\mathscr{P} *(G)$.

Theorem 4 Let $G$ be a finite group. The reduced power graph $\mathscr{P}^{*}(G)$ is regular if and only if $G$ is isomorphic to the cyclic p-group or $\exp (G)=p$, where $p$ is prime.

Proof: Suppose that $\mathscr{P}^{*}(G)$ is regular and let $\langle x\rangle$ be the maximal cyclic subgroup of $G$ of order $n$. It is clear that $\operatorname{deg}_{\mathscr{P} *(G)}(x)=n-2$. Since $\mathscr{P}^{*}(G)$ is regular, we infer that all the maximal cyclic subgroups of $G$ have the same order. Every element of finite group $G$ is in a maximal cyclic subgroup. Hence $\exp (G)=|\langle x\rangle|=n$. By the contradiction, assume that $G$ is not a cyclic $p$-group. Then we can write $n=p_{1}^{a_{1}} p_{2}^{a_{2}} \ldots p_{m}^{a_{m}}$ where $p_{1}<p_{2}<\cdots\left\langle p_{m} m\right\rangle 1$ and $p_{i}^{\prime} s$ are prime. Let $x^{\prime} \in\langle x\rangle$ of order $n / p_{1}$.
We can easily compute the degree of vertex $x^{\prime}$ in $\mathscr{P}^{*}(G)$ : that is, $\left(n / p_{1}\right)-2+k \varphi(n)$ where $k$ is the number of maximal cyclic subgroups which contain $x^{\prime}$ and $\varphi$ is the Eulerian function. Since $\mathscr{P}^{*}(G)$ is regular, $\left(n / p_{1}\right)-2+k \varphi(n)=n-2$ which implies that $k\left(p_{2}-1\right)\left(p_{3}-1\right) \cdots\left(p_{m}-1\right)=p_{2} p_{3} \ldots p_{m}$. Also $p_{2}>2\left(p_{2} \neq 1\right)$. If prime number $q$ divides $p_{2}-1$, then $q \mid p_{2} p_{3} \ldots p_{m}$. Thus $q=p_{i}$ for some $2 \leqslant i \leqslant m$ which is a contradiction. Hence $G$ is a finite $p$ group and $\mathscr{P}^{*}(G)$ is $n-2$ regular. If $n=p$, then $\exp (G)=p$. Suppose that $n=p^{t}>p$. Let $\langle x\rangle$ and $\langle y\rangle$ be distinct maximal cyclic subgroups of order $n$. The regularity $\mathscr{P}^{*}(G)$ implies that these subgroups are disjoint, otherwise the element $g \in\langle x\rangle \cap\langle y\rangle$ has degree greater of $n-2$ in $\mathscr{P}^{*}(G)$.

Suppose the maximal cyclic subgroups of $G$ are not unique. Let $z \in Z(G)$ be of prime order $p$. Then there exists a maximal cyclic subgroup $\langle x\rangle$ such that $z \notin\langle x\rangle$. Now $\langle z x\rangle$ is a maximal cyclic group such that $\langle z x\rangle \neq\langle x\rangle$ and $\langle z x\rangle \cap\langle x\rangle \neq\langle 1\rangle$ which is a contradiction. Hence the maximal cyclic subgroup of $G$ is unique. Thus $G$ is a cyclic group.

\section{CLIQUE NUMBER AND CHROMATIC NUMBER}

It is clear that $\chi(\Gamma) \geqslant \omega(\Gamma)$ for every graph $\Gamma$ and a graph $\Gamma$ is perfect if $\chi\left(\Gamma_{1}\right)=\omega\left(\Gamma_{1}\right)$ for all induced subgraphs $\Gamma_{1}$ of $\Gamma$. From the strong perfect graph theorem given in Ref. 9, a finite graph $\Gamma$ is perfect if and only if neither $\Gamma$ nor $\bar{\Gamma}$ contains an odd cycle of length at least 5 as an induced subgraph. Utilizing this fact, we prove that the power graph of each group is perfect.

Theorem 5 The power graph of a finite group is perfect.

Proof: Suppose that $\mathscr{P}(G)$ contains an induced cycle subgraph $C$ of odd length at least 5 . Let $\vec{C}$ be a related directed subgraph to $C$ in the direct power graph $\overrightarrow{\mathscr{P}}(G)$. Then $\vec{C}$ must have a strong directed path of length two which makes a chordal in $C$ which is a contradiction. Now suppose that $\bar{C}$ is an induced cycle subgraph of odd length at least 5 in $\overline{\mathscr{P}(G)}$. If $\bar{C}$ is a cycle of length 5 , then $C$ is also a cycle of length 5 in $\mathscr{P}(G)$ which is not possible. Now assume that $\bar{C}$ is a cycle of length at least 7 . Then we can easily find a triangle in $C$. On the other hand, we should have indeg $\vec{C}_{\vec{C}}(x)=0$ or outdeg $\overrightarrow{\vec{C}}(x)=0$ for every vertex $x$ in $V(C)$ which is not true for the above triangle. Thus there is no odd cycle of length at least 5 as an induced subgraph in $\overline{\mathscr{P}(G)}$.

Theorem 6 Let $G$ be a finite group. Then

$$
\omega(\mathscr{P}(G))=\max \left\{\omega\left(\mathscr{P}\left(\mathbb{Z}_{n}\right)\right): n \in \pi_{e}(G)\right\} .
$$


Proof: Suppose that $C=\left\{x_{1}, \ldots, x_{m}\right\} \subseteq V(\mathscr{P}(G))$ induces a complete subgraph. Clearly $\langle C\rangle$ is an abelian subgroup. Let $C_{i}=\left\{S_{p_{i}}(\langle x\rangle): x \in C\right\}$, where $S_{p_{i}}(\langle x\rangle)$ is the Sylow $p_{i}$-subgroup of $\langle x\rangle$. Also, let $\left|S_{p_{i}}\left(\left\langle x_{k_{i}}\right\rangle\right)\right|=\max \left\{\left|S_{p_{i}}(\langle x\rangle)\right|: x \in C\right\}$. Since $\left\langle S_{p_{i}}\left(x_{s}\right), S_{p_{i}}\left(x_{t}\right)\right\rangle$ is cyclic for every $1 \leqslant s, t \leqslant m$, it is easy to see that $\left\langle C_{i}\right\rangle=S_{p_{i}}\left(\left\langle x_{k_{i}}\right\rangle\right)$ is a cyclic group. Therefore $\langle C\rangle=\left\langle C_{1}, \ldots, C_{m}\right\rangle$ is a cyclic group and the result follows.

In what follows, we shall give a formula for the clique number of a finite cyclic group.

Lemma 2 Let $n=p_{1}^{\lambda_{1}} \ldots p_{r}^{\lambda_{r}}$ be a natural number and $m=\lambda_{1}+\cdots+\lambda_{r}$. Let $\mathscr{S}$ be the set of all $(m+1)$ tuples $\left(d_{0}, d_{1}, \ldots, d_{m}\right)$ such that $n=d_{0}>d_{1}>\cdots>$ $d_{m}=1$ is a chain of divisors of $n$ and $d_{i-1} / d_{i}$ is a prime for all $i=1, \ldots, m$. Let $f: \mathscr{S} \longrightarrow \mathbb{N}$ be a map defined by $f\left(d_{0}, \ldots, d_{m}\right)=\varphi\left(d_{0}\right)+\cdots+\varphi\left(d_{m}\right)$. Then $f$ takes its maximum value at $\left(d_{0}, \ldots, d_{m}\right)$ if and only if $d_{i-1} / d_{i} \leqslant d_{i} / d_{i+1}$ for all $0<i<m$, and it is unique with this property.

Proof: Suppose on the contrary that there exists $D=$ $\left(d_{0}, \ldots, d_{m}\right) \in \mathscr{S}$ such that $f(D)=\max f(\mathscr{S})$ and $d_{i-1} / d_{i}>d_{i} / d_{i+1}$ for some $i$. Further, we assume that $i$ is a maximum with respect to this property for all $C \in \mathscr{S}$ with $f(C)=\max f(\mathscr{S})$, i.e.,

$$
\begin{gathered}
i=\max \left\{j \text { such that } \frac{c_{j-1}}{c_{j}}>\frac{c_{j}}{c_{j+1}}, \text { for some } C \in \mathscr{S}\right. \\
\text { such that } f(C)=\max f(\mathscr{S})\} .
\end{gathered}
$$

for all $C=\left(c_{0}, \ldots, c_{m}\right)$. Let $p=d_{i-1} / d_{i}$ and $q=$ $d_{i} / d_{i+1}$. If $d_{i}^{\prime}=p d_{i+1}$ and $d_{j}^{\prime}=d_{j}$ whenever $j \neq i$, then

$$
D^{\prime}=\left(d_{0}^{\prime}, d_{1}^{\prime}, \ldots, d_{m}^{\prime}\right) \in \mathscr{S}
$$

and one can see that $f\left(D^{\prime}\right) \geqslant f(D)$ and the equality holds if and only if $p=3, q=2, q \mid d_{i+1}$ and $p \nmid d_{i+1}$. By assumption $f(D)=f\left(D^{\prime}\right)$ and hence $p=3, q=2$, $q \mid d_{i+1}$ and $p \nmid d_{i+1}$. But there exists $j>i$ such that $d_{j-1}^{\prime} / d_{j}^{\prime}>d_{j}^{\prime} / d_{j+1}^{\prime}=2$, which contradicts the choice of $i$. The uniqueness is a direct consequence of the inequalities $d_{i-1} / d_{i} \leqslant d_{i} / d_{i+1}(0<i<m)$, which hold for all $D \in \mathscr{S}$ admitting $f(D)=\max f(\mathscr{S})$. The converse is clear.

Theorem 7 Let $n=p_{1}^{\lambda_{1}} p_{2}^{\lambda_{2}} \ldots p_{r}^{\lambda_{r}}$ with $p_{1}<p_{2}<$ $\cdots<p_{r}$. Then

$$
\omega\left(\mathscr{P}\left(\mathbb{Z}_{n}\right)\right)=\varphi(n)+\varphi\left(\frac{n}{p_{1}}\right)+\cdots+\varphi\left(\frac{n}{p_{1}^{\lambda_{1}}}\right)
$$

$$
\begin{aligned}
& +\varphi\left(\frac{n}{p_{1}^{\lambda_{1}} p_{2}}\right)+\cdots+\varphi\left(\frac{n}{p_{1}^{\lambda_{1}} p_{2}^{\lambda_{2}}}\right) \\
& +\cdots+\varphi\left(\frac{n}{p_{1}^{\lambda_{1}} p_{2}^{\lambda_{2}} \cdots p_{r}}\right)+\cdots \\
& +\varphi\left(\frac{n}{p_{1}^{\lambda_{1}} p_{2}^{\lambda_{2}} \cdots p_{r}^{\lambda_{r}-1}}\right)+\varphi(1)
\end{aligned}
$$

where $\varphi$ is the Eulerian function.

Proof: Let $Y$ be a clique in $\mathscr{P}\left(\mathbb{Z}_{n}\right)$. We first observe that if $y \in V(Y)$, then $r y \in V(Y)$ whenever $\operatorname{gcd}(r,|y|)=1$. Hence the elements of $V(Y)$ can be partitioned into sets each of which contains elements of the same order. Thus $V(Y)=Y_{h_{1}} \cup$ $Y_{h_{2}} \cup \cdots \cup Y_{h_{k}}$, where $Y_{h_{i}}$ possesses of all elements of order $h_{i}$ and $\left|Y_{h_{i}}\right|=\varphi\left(h_{i}\right)$. By Lemma 1 and the fact that $Y$ is a complete subgraph of $\mathscr{P}\left(\mathbb{Z}_{n}\right)$, it follows that for each $i, j \leqslant m$, either $h_{i} \mid h_{j}$ or $h_{j} \mid h_{i}$. Without loss of generality, we assume that $h_{1}|\cdots| h_{k}$. On the other hand, for any chain of positive divisors $l_{1}, \ldots, l_{t}$ of $n$ such that $l_{1}|\cdots| l_{t}$, we can find a clique of size $\sum_{i=1}^{t} \varphi\left(l_{i}\right)$ in $\mathscr{P}\left(\mathbb{Z}_{n}\right)$. Now suppose that $X$ is a maximal clique in $\mathscr{P}\left(\mathbb{Z}_{n}\right)$. Then $V(X)=X_{d_{0}} \cup X_{d_{1}} \cup \cdots \cup X_{d_{m}}\left(d_{0}=n\right)$, where $X_{d_{i}}$ possesses of all elements of order $d_{i},\left|X_{d_{i}}\right|=\varphi\left(d_{i}\right)$, and $d_{m}|\cdots| d_{0}$. Since $X$ is a maximal clique, then $|V(X)|=\sum_{i=0}^{m} \varphi\left(d_{i}\right)=\omega\left(\mathscr{P}\left(\mathbb{Z}_{n}\right)\right)$. Hence by Lemma $2, m=\lambda_{1}+\cdots+\lambda_{r}$ and $d_{i}=\max \{h: h \mid$ $\left.d_{i-1}, h<d_{i-1}\right\}$.

The following corollary gives a shortened formula for $\omega\left(\mathscr{P}\left(\mathbb{Z}_{n}\right)\right)$.

Corollary 2 Let $n=p_{1}^{\lambda_{1}} p_{2}^{\lambda_{2}} \ldots p_{r}^{\lambda_{r}}$ such that $p_{1}<$ $p_{2}<\ldots<p_{r}$. Then

$$
\omega\left(\mathscr{P}\left(\mathbb{Z}_{n}\right)\right)=\sum_{i=2}^{r+1} \varphi\left(\frac{n}{p_{1}^{\lambda_{1}} p_{2}^{\lambda_{2}} \ldots p_{i-1}^{\lambda_{i-1}}}\right) p_{i-1}^{\lambda_{i-1}-1}+\varphi(n) .
$$

Proof:

$$
\begin{gathered}
\omega\left(\mathscr{P}\left(\mathbb{Z}_{n}\right)\right)=\varphi(1)+\varphi\left(p_{r}\right)+\cdots+\varphi\left(p_{r}^{\lambda_{r}-1}\right) \\
+\varphi\left(p_{r}^{\lambda_{r}}\right)\left(\varphi(1)+\varphi\left(p_{r-1}\right)+\cdots+\varphi\left(p_{r-1}^{\lambda_{r-1}-1}\right)\right) \\
+\varphi\left(p_{r}^{\lambda_{r}} p_{r-1}^{\lambda_{r-1}}\right)\left(\varphi(1)+\varphi\left(p_{r-2}\right) \cdots+\varphi\left(p_{r-2}^{\lambda_{r-2}-1}\right)\right) \\
+ \\
\vdots \\
+\varphi\left(p_{r}^{\lambda_{r}} \ldots p_{2}^{\lambda_{2}}\right)\left(\varphi(1)+\varphi\left(p_{1}\right)+\cdots+\varphi\left(p_{1}^{\lambda_{1}-1}\right)\right) \\
+\varphi(n) .
\end{gathered}
$$


Thus

$$
\begin{aligned}
\omega\left(\mathscr{P}\left(\mathbb{Z}_{n}\right)\right)= & p_{r}^{\lambda_{r}-1}+\varphi\left(p_{r}^{\lambda_{r}}\right) p_{r-1}^{\lambda_{r-1}-1} \\
& +\varphi\left(p_{r}^{\lambda_{r}} p_{r-1}^{\lambda_{r-1}}\right) p_{r-2}^{\lambda_{r-2}-1} \\
& +\cdots+\varphi\left(p_{r}^{\lambda_{r}} p_{r-1}^{\lambda_{r-1}} \ldots p_{2}^{\lambda_{2}}\right) p_{1}^{\lambda_{1}-1}+\varphi(n) .
\end{aligned}
$$

Let $n_{i}=p_{i}^{\lambda_{i}} p_{i+1}^{\lambda_{i+1}} \ldots p_{r}^{\lambda_{r}}$ and $n_{r+1}=1$. Then

$$
\begin{aligned}
\omega\left(\mathscr{P}\left(\mathbb{Z}_{n}\right)\right)= & \sum_{i=2}^{r+1} \varphi\left(n_{i}\right) p_{i-1}^{\lambda_{i-1}-1}+\varphi(n) \\
= & \sum_{i=2}^{r+1} \varphi\left(\frac{n}{p_{1}^{\lambda_{1}} p_{2}^{\lambda_{2}} \ldots p_{i-1}^{\lambda_{i-1}}}\right) p_{i-1}^{\lambda_{i-1}-1} \\
& +\varphi(n) .
\end{aligned}
$$

Corollary 3 Let $n=p_{1}^{\lambda_{1}} p_{2}^{\lambda_{2}} \ldots p_{r}^{\lambda_{r}}$ such that $p_{1}<$ $p_{2}<\cdots<p_{r}$. Then

$$
\begin{aligned}
\chi\left(\mathscr{P}\left(\mathbb{Z}_{n}\right)\right)=\sum_{i=2}^{r+1} \varphi\left(\frac{n}{p_{1}^{\lambda_{1}} p_{2}^{\lambda_{2}} \ldots p_{i-1}^{\lambda_{i-1}}}\right) p_{i-1}^{\lambda_{i-1}-1} \\
+\varphi(n) .
\end{aligned}
$$

Proof: By Theorem 5 and Corollary 2, the result is clear.

\section{AUTOMORPHISM GROUP OF POWER GRAPH OF $\mathscr{P}\left(\mathbb{Z}_{n}\right)$}

In Ref. 10, Cameron and Ghosh showed that the only finite group whose automorphism group is the same as that of its power graph is the Klein four group. In this section, we discuss the automorphism group of $\mathscr{P}\left(\mathbb{Z}_{n}\right)$. For any graph $\Gamma$ and $v \in V(\Gamma)$, the set of neighbours of the vertex $v$ in $\Gamma$ is denoted by $N_{\Gamma}(v)$, or briefly by $N(v)$. Furthermore, we define $N[v]=N(v) \cup\{v\}$.

Lemma 3 If $x, y \in V\left(\mathscr{P}\left(\mathbb{Z}_{n}\right)\right)$ and $|x|=|y|$, then $N[x]=N[y]$.

Proof: As $\mathbb{Z}_{n}$ has a unique subgroup of order $|x|$, we have $\langle x\rangle=\langle y\rangle$ and the proof is straightforward.

Now let $\left\{d_{1}, d_{2}, \ldots, d_{r}\right\}$ be the set of all divisors of $n$ other than 1 and $n$. Let $A_{0}=\left\{g \in \mathbb{Z}_{n}:|g|=n\right\} \cup\{\overline{0}\}$ and $A_{i}=\left\{g \in \mathbb{Z}_{n}:|g|=d_{i}\right\}$, for $i=1,2, \ldots, r$. Then by Lemma 1 , the induced subgraphs of $\mathscr{P}\left(\mathbb{Z}_{n}\right)$ on $A_{i}$ 's are complete of order $\varphi\left(d_{i}\right)$, for $i=1,2 \ldots, r$ and the induced subgraph of $\mathscr{P}\left(\mathbb{Z}_{n}\right)$ on $A_{0}$ is a complete graph of order $\varphi(n)+1$. Hence, by Lemma 3 , every bijection $\alpha: \mathbb{Z}_{n} \longrightarrow \mathbb{Z}_{n}$ such that $\left.\alpha\right|_{A_{i}}: A_{i} \longrightarrow A_{i}$ is a permutation for $i=0,1,2, \ldots, r$, is an automorphism of $\mathscr{P}\left(\mathbb{Z}_{n}\right)$. Thus we have the following theorem.
Theorem 8 Aut $\left(\mathscr{P}\left(\mathbb{Z}_{n}\right)\right)$ has a subgroup isomorphic to $S_{\varphi(n)+1} \times \prod_{d \mid n, d \neq 1, n} S_{\varphi(d)}$.

Corollary 4 Let $n$ be a natural number such that for every $x, y \in \mathbb{Z}_{n}, \operatorname{deg}(x) \neq \operatorname{deg}(y)$ whenever $|x| \neq|y|$. Then

$$
\operatorname{Aut}\left(\mathscr{P}\left(\mathbb{Z}_{n}\right)\right) \cong S_{\varphi(n)+1} \times \prod_{d \mid n, d \neq 1, n} S_{\varphi(d)} .
$$

Proof: If $\alpha \in \operatorname{Aut}\left(\mathscr{P}\left(\mathbb{Z}_{n}\right)\right)$ and $|x| \neq|y|$, then $\alpha(x) \neq$ $y$. Hence $\alpha\left(A_{i}\right)=A_{i}$, for $i=0,1,2, \ldots, r$, where $A_{i}$ 's are defined as above. Thus $\alpha \in S_{A_{0}} \times S_{A_{1}} \times \cdots \times$ $S_{A_{r}}$.

As an example, if $G=\mathbb{Z}_{12}$ and $x=\overline{2}, y=\overline{6}$ are elements of $G$. Then $|x|=6$ and $|y|=2$, and $\operatorname{deg}(x)=\operatorname{deg}(y)=9$. However, there is no automorphism of $\mathscr{P}\left(\mathbb{Z}_{n}\right)$ which maps $x$ to $y$. Hence we have $\operatorname{Aut}\left(\mathscr{P}\left(\mathbb{Z}_{12}\right)\right) \cong S_{\varphi(12)+1} \times \prod_{d \mid 12, d \neq 1,12} S_{\varphi(d)}$. The same happens for $\mathbb{Z}_{24}$.

We state the following conjecture for $\operatorname{Aut}\left(\mathscr{P}\left(\mathbb{Z}_{n}\right)\right)$. In spite the fact that we have much evidence for some small values of $n$ and strongly believe that it is true for all $n$ having at least two distinct prime divisors, we have not able to prove it completely as yet.

Conjecture 1 For every natural number n,

$$
\operatorname{Aut}\left(\mathscr{P}\left(\mathbb{Z}_{n}\right)\right) \cong S_{\varphi(n)+1} \times \prod_{d \mid n, d \neq 1, n} S_{\varphi(d)} .
$$

Finally, we compute the degree of all vertices in $\mathscr{P}\left(\mathbb{Z}_{n}\right)$.

Theorem 9 The degree of an arbitrary vertex $x$ in $\mathscr{P}\left(\mathbb{Z}_{n}\right)$ is

$$
\operatorname{deg}(x)=d-1+\sum_{r d \mid n, r \geqslant 2} \varphi(r d),
$$

where $d$ is the order of $x$ in $\mathbb{Z}_{n}$.

Proof: By Lemma 1, the number of elements of order $r d$ in $\mathbb{Z}_{n}$ is $\varphi(r d)$. The result follows immediately.

We may give a similar formula for the vertex degrees of $\mathscr{P}\left(\mathbb{Z}_{n}\right)$ as follows.

Corollary 5 Let $x$ be a vertex in $\mathscr{P}\left(\mathbb{Z}_{n}\right)$. Then

$$
\operatorname{deg}(x)=d-1-\varphi(d)+\varphi(d) \sigma(m) \frac{n}{d m},
$$

where $d$ is the order of $x$ in $\mathbb{Z}_{n}, m=\prod_{p \mid d, p^{k} \| n / d} p^{k}$ and $\sigma(m)$ is the sum of divisors $m$. Also, $p^{k} \| n / d$ means that $\frac{n}{d} / p^{k}$ does not have divisor $p$. 
Proof: By Theorem 9, we have

$$
\operatorname{deg}(x)=d-1+\sum_{r>1, r \mid n / d} \varphi(r d) .
$$

Assume $n=p_{1}^{a_{1}} p_{2}^{a_{2}} \ldots p_{r_{r}}^{a_{r}}$ and $d=p_{1}^{b_{1}} p_{2}^{b_{2}} \ldots p_{r}^{b_{r}}$. Also, let $r_{x}=\prod_{b_{i} \neq 0} p_{i}^{c_{i}}$ for each divisor $r=$ $p_{1}^{c_{1}} p_{2}^{c_{2}} \ldots p_{r}^{c_{r}}$ of $n$. Then

$$
\begin{aligned}
\varphi(r d) & =\prod_{b_{i} \neq 0} p_{i}^{b_{i}+c_{i}-1}\left(p_{i}-1\right) \prod_{b_{i}=0, c_{i} \neq 0} p_{i}^{c_{i}-1}\left(p_{i}-1\right) \\
& =\prod_{b_{i} \neq 0} p_{i}^{c_{i}} \prod_{b_{i} \neq 0} p_{i}^{b_{i}-1}\left(p_{i}-1\right) \prod_{b_{i}=0, c_{i} \neq 0} p_{i}^{c_{i}-1}\left(p_{i}-1\right) \\
& =r_{x} \varphi(d) \varphi\left(\frac{r}{r_{x}}\right) .
\end{aligned}
$$

Hence

$$
\begin{aligned}
\sum_{r \mid n / d} \varphi(r d) & =\sum_{r \mid n / d} r_{x} \varphi(d) \varphi\left(\frac{r}{r_{x}}\right) \\
& =\varphi(d) \sum_{r \mid n / d} r_{x} \varphi\left(\frac{r}{r_{x}}\right) \\
& =\varphi(d) \sum_{\substack{r_{x}\left|n_{x}^{\prime} \\
\left(r / r_{x}\right)\right| n^{\prime} / n_{x}^{\prime}}} r_{x} \varphi\left(\frac{r}{r_{x}}\right) \\
& =\varphi(d)\left(\sum_{r_{x} \mid n_{x}^{\prime}} r_{x}\right)\left(\sum_{\left(r / r_{x}\right) \mid n^{\prime} / n_{x}^{\prime}} \varphi\left(\frac{r}{r_{x}}\right)\right) \\
& =\varphi(d) \sigma\left(n_{x}^{\prime}\right) \frac{n^{\prime}}{n_{x}^{\prime}},
\end{aligned}
$$

where $n^{\prime}=n / d$.

\section{REFERENCES}

1. Bosak J (1963) The graphs of semigroups. In: Fiedler M (ed) Theory of Graphs and its Applications: Proceedings of the Symposium held in Smolenice in June 1963, pp 119-25.

2. Kelarev AV, Quinn SJ (2000) A combinatorial property and power graphs of groups. In: Contributions to General Algebra, vol 12, pp 229-35.

3. Kelarev AV, Quinn SJ (2002) Directed graph and combinatorial properties of semigroups. J Algebra 251, 16-26.

4. Chakrabarty I, Ghosh Sen MK (2009) Undirected power graphs of semigroups. Semigroup Forum 78, 410-26.

5. Cameron PJ (2010) The power graph of a finite group II. J Group Theor 13, 779-83.

6. Roland S (1994) Subgroup Lattices of Groups, 2nd edn, Walter de Gruyter, Berlin, pp 12-3.

7. Kuratowski K (1930) Sur le problème des courbes gauches en topologie. Fund Math 15, 271-83.
8. Lytkina DV (2007) Structure of a group with elements of order at most 4. Siberian Math J 48, 283-7.

9. Chudnovsky M, Robertson N, Seymour P, Thomas R (2006) The strong perfect graph theorem. Ann Math 164, 51-229.

10. Cameron PJ, Ghosh S (2011) The power graph of a finite group. Discrete Math 311, 1220-2. 